\title{
Evolución de las propiedades mecánicas durante la cristalización de
}

\section{cintas amorfas $\mathrm{Mg}-\mathrm{Ni}$}

\author{
P. Pérez*, G. Garcés*, G. Caruana* y P. Adeva*
}

\begin{abstract}
Resumen En este trabajo se estudia la evolución de las propiedades mecánicas con la temperatura, mediante ensayos de tracción, de dos aleaciones amorfas Mg-23Ni y Mg-35Ni (\% en masa) procesadas por solidificación rápida. Las dos aleaciones experimentan, en el intervalo de temperaturas de ensayo (temperatura ambiente- $350^{\circ} \mathrm{C}$ ), procesos de cristalización que transcurren en dos etapas, en el caso de la aleación $\mathrm{Mg}-23 \mathrm{Ni}$, y en una etapa, en la aleación $\mathrm{Mg}-35 \mathrm{Ni}$. Los cambios microestructurales asociados a los procesos de cristalización son responsables de la variación de las propiedades mecánicas con la temperatura así como de las diferencias observadas entre las dos aleaciones.
\end{abstract}

Palabras clave Aleaciones de magnesio. Amorfos. Propiedades mecánicas.

\section{Evolution of mechanical properties during crystallization of amorphous $\mathrm{Mg}-\mathrm{Ni}$ ribbons}

Abstract

\begin{abstract}
The evolution of mechanical properties with temperature of rapidly solidified $\mathrm{Mg} 23 \mathrm{Ni}$ and Mg-35Ni (mass \%) have been studied by tensile tests. Both alloys crystallise in the test temperature range (room temperature- $350^{\circ} \mathrm{C}$ ). The process takes place in two steps for $\mathrm{Mg} 23 \mathrm{Ni}$ and in one step for $\mathrm{Mg} 35 \mathrm{Ni}$. The microstructural changes in the alloys by the crystallisation processes are responsible for mechanical properties evolution with temperature as well as for differences observed between both alloys
\end{abstract}

Keywords Magnesium alloys. Amorphous. Mechanical properties.

\section{INTRODUCCIÓN}

Las aleaciones de magnesio son materiales estructurales muy prometedores debido a su baja densidad y elevada resistencia específica. Además, su bajo coste y las grandes reservas existentes las hacen ventajosas respecto a otras aleaciones en aplicaciones aeronáuticas y en el sector del automó$\mathrm{vil}^{[1]}$. Los principales inconvenientes que presentan estas aleaciones son sus limitaciones de resistencia mecánica y su baja resistencia a la corrosión. Se sabe que ambas propiedades mejoran claramente si las aleaciones tienen una microestructura amorfa o nanocristalina. Por ello, desde la década de los 90 se están realizando un gran número de investigaciones encaminadas a desarrollar y sintetizar sistemas de aleaciones de magnesio amorfas en un amplio intervalo de composiciones $^{[2 \text { y } 3]}$, así como a estudiar su estabilidad térmica

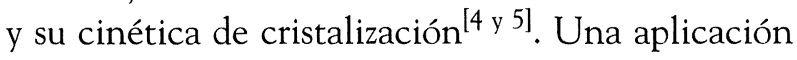

reciente de las aleaciones amorfas y nanocristalinas de magnesio, concretamente las $\mathrm{Mg}-\mathrm{Ni}$, es para la fabricación de electrodos negativos de baterías recargables debido a su gran capacidad de almacenar hidrógeno como hidruros. Por ello, hay bastantes grupos estudiando aleaciones amorfas y nanocristalinas $\mathrm{Mg}-\mathrm{Ni}$ con adiciones de tierras raras ${ }^{[4}$ y $\left.6-9\right]$.

Las propiedades mecánicas se han estudiado fundamentalmente en amorfos masivos, mediante ensayos de compresión ${ }^{[3]}$. Los datos disponibles sobre las propiedades mecánicas de cintas obtenidas por solidificación rápida son, únicamente, a partir de medidas de dureza ${ }^{[6]}$, de modo que no se dispone de un conocimiento completo de la respuesta mecánica del material a diferentes temperaturas según sea su estado: amorfo, parcialmente cristalino o nanocristalino.

Este estudio tiene como objetivo la caracterización mecánica, mediante ensayos de tracción desde temperatura ambiente $(\mathrm{Ta})$ hasta $350{ }^{\circ} \mathrm{C}$, de

(*) Centro Nacional de Investigaciones Metalúrgicas (CENIM), Avda. Gregorio del Amo, 8. 28040 Madrid, España. 
cintas amorfas de dos aleaciones $\mathrm{Mg}-\mathrm{Ni}$ de composición eutéctica e hipereutéctica. Además, se analiza la variación de las propiedades mecánicas con la microestructura, dado que ésta evoluciona desde el estado amorfo hasta el cristalino por efecto de la temperatura de ensayo.

\section{MATERIALES Y PROCEDIMIENTO EXPERIMEN- TAL}

Las cintas de dos aleaciones de composición eutéctica $\mathrm{Mg}-23 \mathrm{Ni}$ e hipereutéctica $\mathrm{Mg}-35 \mathrm{Ni}$ (\% en masa) fueron suministradas por el Prof. Sommer, del Max-Planck Institute ${ }^{[10]}$. Las cintas se procesaron por la técnica de la rueda empleando atmósfera de helio y rueda de cobre. Las dimensiones de las cintas son $39 \mu \mathrm{m}$ de espesor y $0,7 \mathrm{~mm}$ de anchura en el caso de la aleación $\mathrm{Mg} 23 \mathrm{Ni}$ y $59 \mu \mathrm{m}$ de espesor y $1,18 \mathrm{~mm}$ de anchura en el caso de la $\mathrm{Mg} 35 \mathrm{Ni}$. Los dos materiales presentaban un buen estado superficial y podían doblarse $180^{\circ}$.

Los ensayos de tracción se realizaron utilizando mordazas planas, una celda de carga de $200 \mathrm{~N}$ y una sensibilidad de 0,1 N. El sistema de calentamiento, acoplado a las mordazas, permite realizar ensayos hasta $500^{\circ} \mathrm{C}$ en atmósfera controlada. Los ensayos desde temperatura ambiente hasta $350^{\circ} \mathrm{C}$ se realizaron a $10^{-2} \mathrm{~Pa}$ utilizando una longitud de probeta de $10 \mathrm{~mm}$ y una velocidad inicial de deformación de $10^{-4} \mathrm{~s}^{-1}$. Se emplearon siempre tiempos de calentamiento de unos 6 min hasta alcanzar la temperatura de ensayo.

La microestructura y las superficies de fractura de las cintas se caracterizaron mediante Microscopía Electrónica de Barrido y de Transmisión. La preparación de muestras para caracterizar la micro-

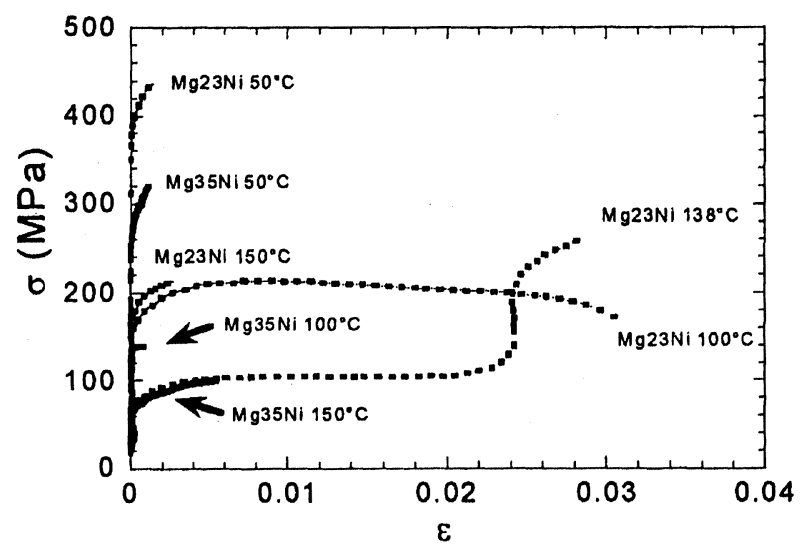

estructura se realizó mediante adelgazamiento iónico.

\section{RESULTADOS}

El comportamiento en tracción de las cintas de las dos aleaciones ensayadas, desde temperatura ambiente hasta $350^{\circ} \mathrm{C}$, se muestra en las figuras 1 y 2 . Las curvas de las dos aleaciones muestran un cambio de comportamiento con la temperatura de ensayo. Ambas aleaciones presentan elevados valores de resistencia en el intervalo $\mathrm{Ta}-100{ }^{\circ} \mathrm{C}$ aunque son siempre superiores los de la aleación $\mathrm{Mg} 23 \mathrm{Ni}$. Los máximos valores los alcanzan a $50^{\circ} \mathrm{C}$, siendo de $450 \mathrm{MPa}$ en el caso del $\mathrm{Mg} 23 \mathrm{Ni}$ y $325 \mathrm{MPa}$, en el caso del Mg35Ni. En este intervalo de temperaturas los materiales son muy frágiles aunque la aleación eutéctica presenta un alargamiento del $3 \%$ a $100^{\circ} \mathrm{C}$.

La microestructura de las dos aleaciones en este intervalo de temperaturas corresponde a la de un material amorfo lo cual es corroborado por el aspecto de sus superficies de fractura (Fig.2) que es del tipo "en venas", típica de un material amorfo.

A temperaturas de deformación entre 138 y $150^{\circ} \mathrm{C}$, la cinta $\mathrm{Mg} 23 \mathrm{Ni}$ experimenta un cambio de comportamiento mecánico. Así, la curva tensión real-deformación real, a $138^{\circ} \mathrm{C}$, muestra dos regímenes. En el primero, la cinta alcanza un estado estacionario a unos $100 \mathrm{MPa}$ y tras una deformación del $2 \%$ la tensión aumenta rápidamente alcanzando un segundo periodo elástico con una deformación plástica muy pequeña. A $150^{\circ} \mathrm{C}$, el material experimenta un aumento del límite elástico, pero rompe nada más sobrepasarlo. Mediante microscopía se ha observado que, durante el

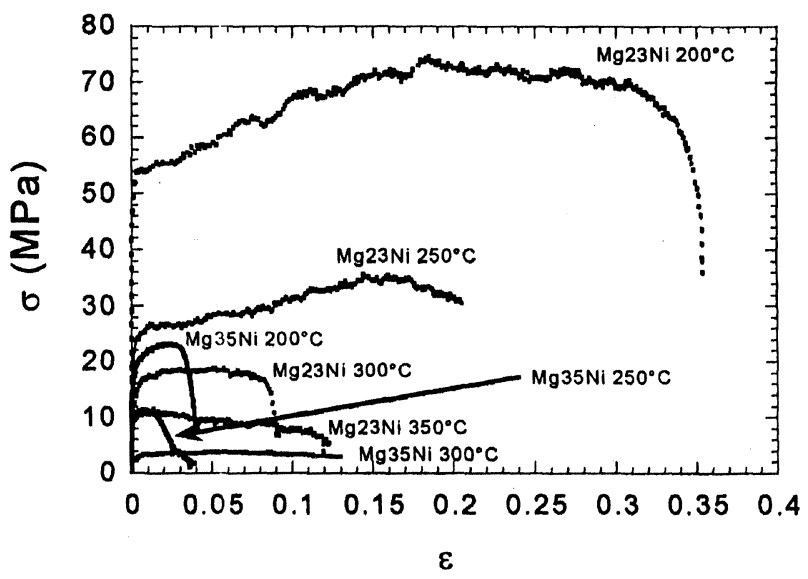

Figura 1. Curvas tensión real-deformación real del Mg23Ni y Mg35Ni entre Ta-350 ${ }^{\circ} \mathrm{C}$.

Figure 1. True stress-true strain curves of $\mathrm{Mg} 23 \mathrm{Ni}$ and $\mathrm{Mg} 35 \mathrm{Ni}$ from $\mathrm{RT}-350^{\circ} \mathrm{C}$. 
Evolución de las propiedades mecánicas durante la cristalización de cintas amorfas $\mathrm{Mg}-\mathrm{Ni}$

P. PÉrez, G. Garcés, G. Caruana y P. AdeVa

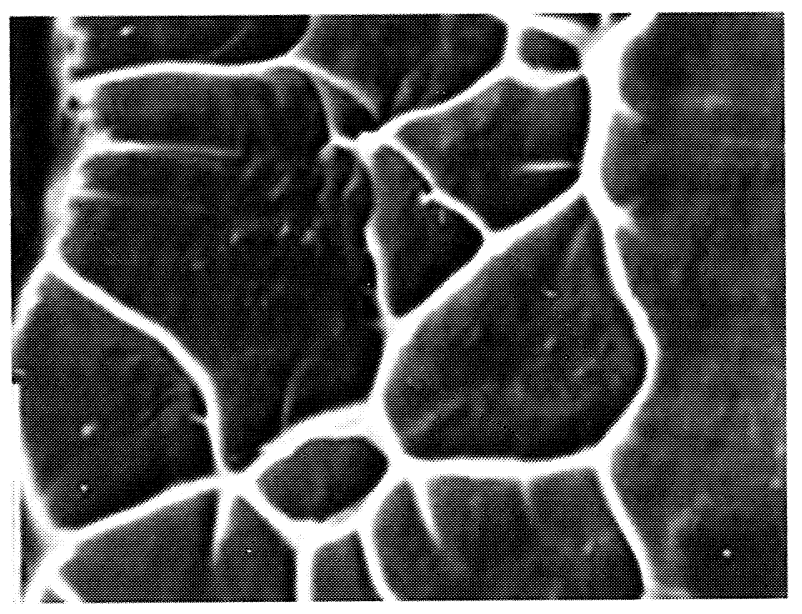

Figura 2. $\mathrm{Mg} 23 \mathrm{Ni}$. Fractura a $100^{\circ} \mathrm{C}$.

Figure 2. $\mathrm{Mg} 23 \mathrm{Ni}$. Fracture at $100^{\circ} \mathrm{C}$.

calentamiento de la cinta hasta la temperatura de ensayo de $150^{\circ} \mathrm{C}$, se forman cristales de magnesio en una matriz nanocristalina. Esta estructura, prácticamente no evoluciona durante el ensayo. La figura 3 presenta la microestructura nanocristalina de la cinta tras el ensayo de tracción a $150^{\circ} \mathrm{C}$. La superficie de fractura de la figura 4 indica, también, que se ha producido la cristalización del material. Por encima de $150^{\circ} \mathrm{C}$, el material experimenta una disminución importante del límite elástico y de la resistencia mecánica con la temperatura de ensayo

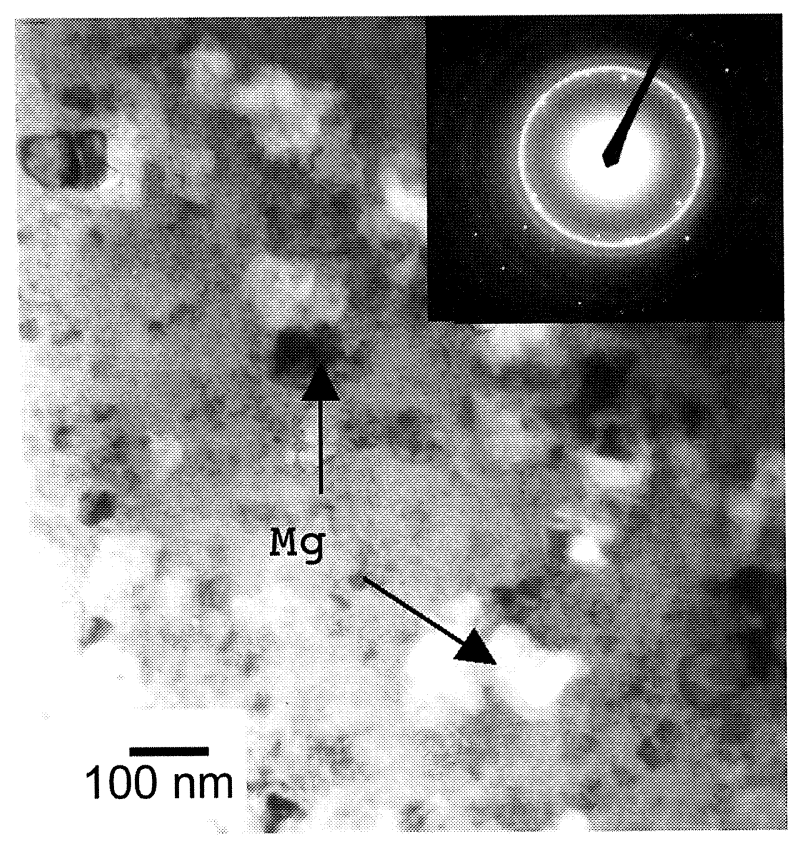

Figura 3. $\mathrm{Mg} 23 \mathrm{Ni}$ ensayado a $150^{\circ} \mathrm{C}$. Imagen de campo claro y diagrama de difracción de electrones mostrando una matriz nanocristalina.

Figure 3. $\mathrm{Mg} 23 \mathrm{Ni}$ tested at $150^{\circ} \mathrm{C}$ Bright field image and diffraction pattern showing a nanocrystalline matrix.

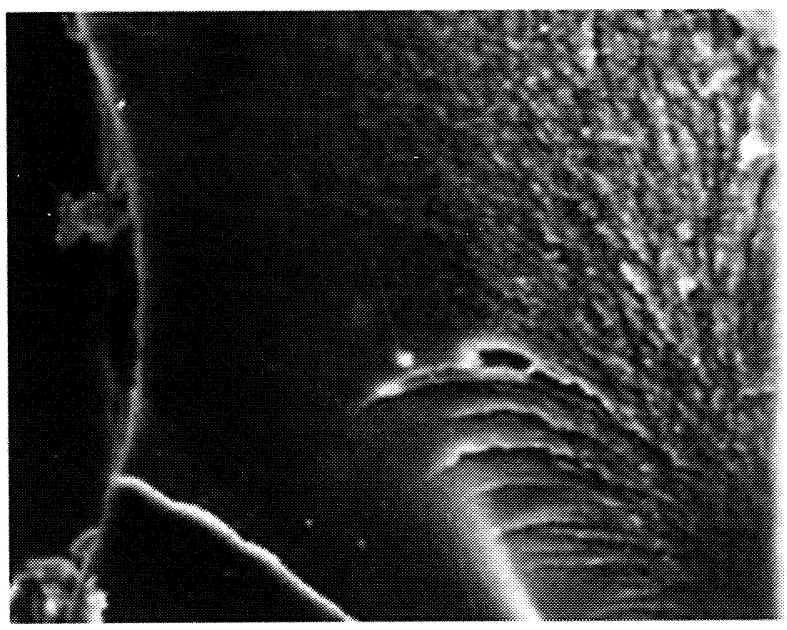

Figura 4. $\mathrm{Mg} 23 \mathrm{Ni}$. Fractura a $150^{\circ} \mathrm{C}$.

Figure 4. $\mathrm{Mg} 23 \mathrm{Ni}$ Fracture at $150^{\circ} \mathrm{C}$.

pero la ductilidad aumenta considerablemente hasta $200{ }^{\circ} \mathrm{C}$. A esta temperatura, la cinta alcanza alargamientos de hasta el $30 \%$. Para temperaturas de ensayo superiores, todas las propiedades mecánicas decrecen, incluido el alargamiento.

La evolución del comportamiento mecánico de la cinta $\mathrm{Mg} 35 \mathrm{Ni}$ con la temperatura, por encima de $100^{\circ} \mathrm{C}$, temperaturas en las que los procesos de cristalización tienen lugar, no presenta cambios respecto a temperaturas más bajas. Sigue mostrando una disminución del límite elástico y de la resistencia, alcanzando niveles muy bajos de ambas propiedades a partir de $150^{\circ} \mathrm{C}$. La ductilidad, sin embargo, aumenta ligeramente.

Es importante destacar que las propiedades mecánicas de esta aleación son siempre inferiores a las de la aleación eutéctica. Este diferente comportamiento debe estar relacionado con las diferencias microestructurales entre las dos aleaciones, tanto durante la cristalización como tras ella.

Comparando las figuras 4 y 5 se deduce que la cinta $\mathrm{Mg} 23 \mathrm{Ni}$ deformada a $150{ }^{\circ} \mathrm{C}$ presenta una microestructura mucho más fina que la cinta Mg35Ni. El microanálisis realizado sobre un área de la figura 5 indica que los cristales claros corresponden a la fase $\mathrm{Mg}_{2} \mathrm{Ni}$ y la fase oscura está constituida por $\mathrm{Mg}$ y pequeños cristales de $\mathrm{Mg}_{2} \mathrm{Ni}$. A elevadas temperaturas, sin embargo, las dos cintas presentan una microestructura completamente cristalizada y de tamaño relativamente grueso lo que conduce a una disminución muy importante de la resistencia. En las micrografías de la figura 6 se muestran las microestructuras de las aleaciones antes de ser ensayadas a $300^{\circ} \mathrm{C}$ y tras ser deformadas. Ambas aleaciones presentan, previo al inicio 


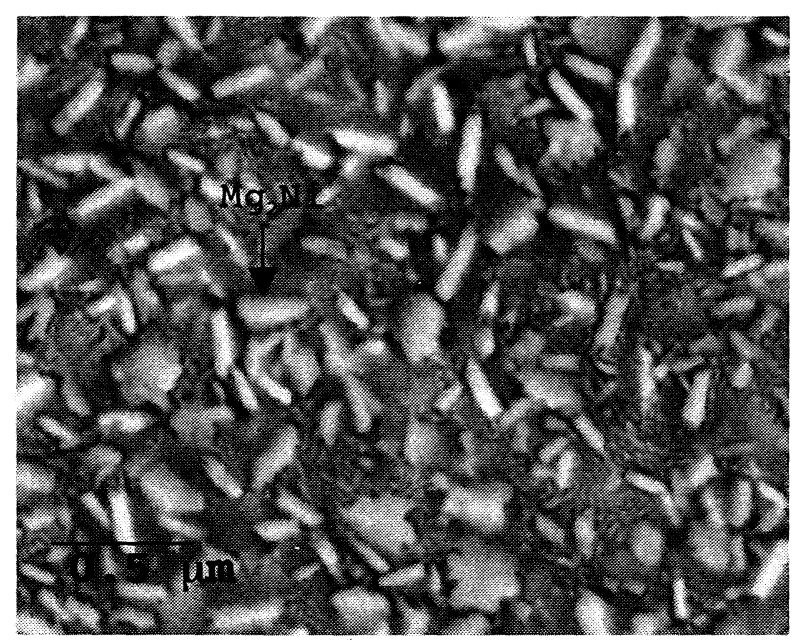

Figura 5. $\mathrm{Mg} 35 \mathrm{Ni}$ ensayado a $150^{\circ} \mathrm{C}$. Imagen de electrones retrodispersados mostrando una estructura completamente cristalizada.

Figure 5. Mg35Ni tested at $150{ }^{\circ} \mathrm{C}$. Backscatterd electron image showing the crystallised structure.

del ensayo, una microestructura bifásica constituida por $\mathrm{Mg}$ y $\mathrm{Mg}_{2} \mathrm{Ni}$. Su morfología, sin embargo, es bien diferente ya que mientras que la aleación Mg23Ni presenta una estructura eutéctica laminar, en la aleación $\mathrm{Mg} 35 \mathrm{Ni}$ las fases son equiaxiales (Figs. 6a) y 6c)). Las microestructuras de las aleaciones tras el ensayo de tracción a $300^{\circ} \mathrm{C}$ son similares, como indican las imágenes de campo claro de las figuras $6 \mathrm{~b}$ ) y $6 \mathrm{~d}$ ) aunque la aleación con más contenido en níquel presenta una mayor fracción de volumen de cristales de la fase intermetálica $\mathrm{Mg}_{2} \mathrm{Ni}$, de acuerdo con su posición en el diagrama de equilibrio.

\section{DISCUSION}

El estudio del comportamiento en tracción de las cintas amorfas $\mathrm{Mg} 23 \mathrm{Ni}$ y $\mathrm{Mg} 35 \mathrm{Ni}$ indica que las propiedades mecánicas son muy sensibles a los procesos de cristalización que suceden en el intervalo de temperaturas de ensayo. Además, se ha comprobado que el estado amorfo es el que proporciona al material la resistencia mecánica más elevada. El aumento del alargamiento que experimenta el material en estado amorfo conforme aumenta la temperatura, alcanzando un estado estacionario, la aleación de $23 \% \mathrm{Ni}$ a $100{ }^{\circ} \mathrm{C}$, se puede asociar a procesos de relajación estructural del amorfo.

La aparición, en la aleación eutéctica a $138^{\circ} \mathrm{C}$, de dos regímenes elásticos y un aumento de la resistencia acompañado de un fenómeno de fragilización se debe al inicio de la cristalización. De acuerdo con los estudios cinéticos de cristalización realizados por Kempen et al. ${ }^{[10]}$ mediante calorimetría isocrona, la aleación $\mathrm{Mg} 23 \mathrm{Ni}$ cristaliza en dos etapas. En la primera, que ocurre alrededor de $164^{\circ} \mathrm{C}$, cristaliza la fase magnesio y la fase metaestable $\mathrm{Mg}_{5,5} \mathrm{Ni}$. En la segunda etapa, a $190{ }^{\circ} \mathrm{C}$, la fase metaestable se descompone en las de equilibrio $\mathrm{Mg}+\mathrm{Mg}_{2} \mathrm{Ni}$. En este trabajo, estos procesos parecen ocurrir a temperaturas inferiores, lo cual es de esperar ya que las condiciones de calentamiento hasta la temperatura de ensayo son diferentes que las que se utilizan en ensayos de calorimetría. Por lo tanto, la primera etapa de cristalización es la que tiene lugar durante los ensayos mecánicos en el intervalo $138-150^{\circ} \mathrm{C}$. En la figura 4 se observa la fase magnesio y una matriz nanocristalina que debe corresponder a la fase metaestable $\mathrm{Mg}_{5,5} \mathrm{Ni}^{[4 \text { y 9] }}$. Kempen et al. ${ }^{[4]}$ observaron que este material contenía una fracción en volumen de la fase metaestable del $70 \%$, tras un recocido de $4 \mathrm{~h}$ a $127{ }^{\circ} \mathrm{C}$. El hecho de que, a $150^{\circ} \mathrm{C}$ se produzca un aumento del límite elástico permite suponer que la fase metaestable $\mathrm{Mg}_{5,5} \mathrm{Ni}$ es muy resistente y que su transformación en las de equilibrio causa la disminución, observada a $200^{\circ} \mathrm{C}$, de la resistencia mecánica del material. La lenta descomposición de la fase $\mathrm{Mg}_{5,5} \mathrm{Ni}$ por debajo de $200^{\circ} \mathrm{C}$ explica la también lenta disminución de la resistencia mecánica en el intervalo 150 $200^{\circ} \mathrm{C}$. Este efecto va acompañado de un aumento en el alargamiento que se mantiene mientras que la microestructura es nanocristalina, alcanzando el máximo del $30 \%$ a $200{ }^{\circ} \mathrm{C}$. Por encima de $200{ }^{\circ} \mathrm{C}$, la fase metaestable que aún queda se descompone más rápidamente y se produce una disminución más importante de la resistencia. Además, conforme aumenta la temperatura de ensayo, la microestructura engrosa y cambia su morfología (Figs. 6a) y 6b)) lo que explica la pérdida notable de propiedades.

La evolución de las propiedades mecánicas con la temperatura de la aleación $\mathrm{Mg} 35 \mathrm{Ni}$ es algo distinta. A diferencia de la aleación eutéctica, este material siempre experimenta, conforme aumenta la temperatura de ensayo, una disminución de resistencia acompañada por un pequeño aumento de la plasticidad. Se puede concluir que la resistencia mecánica de esta aleación disminuye conforme el material tiende hacia la estructura estable que es la cristalina. Las diferencias en comportamiento mecánico, en el intervalo $\mathrm{Ta}-200{ }^{\circ} \mathrm{C}$, entre ambas aleaciones se explican de acuerdo con las diferencias en los procesos de cristalización. La aleación 

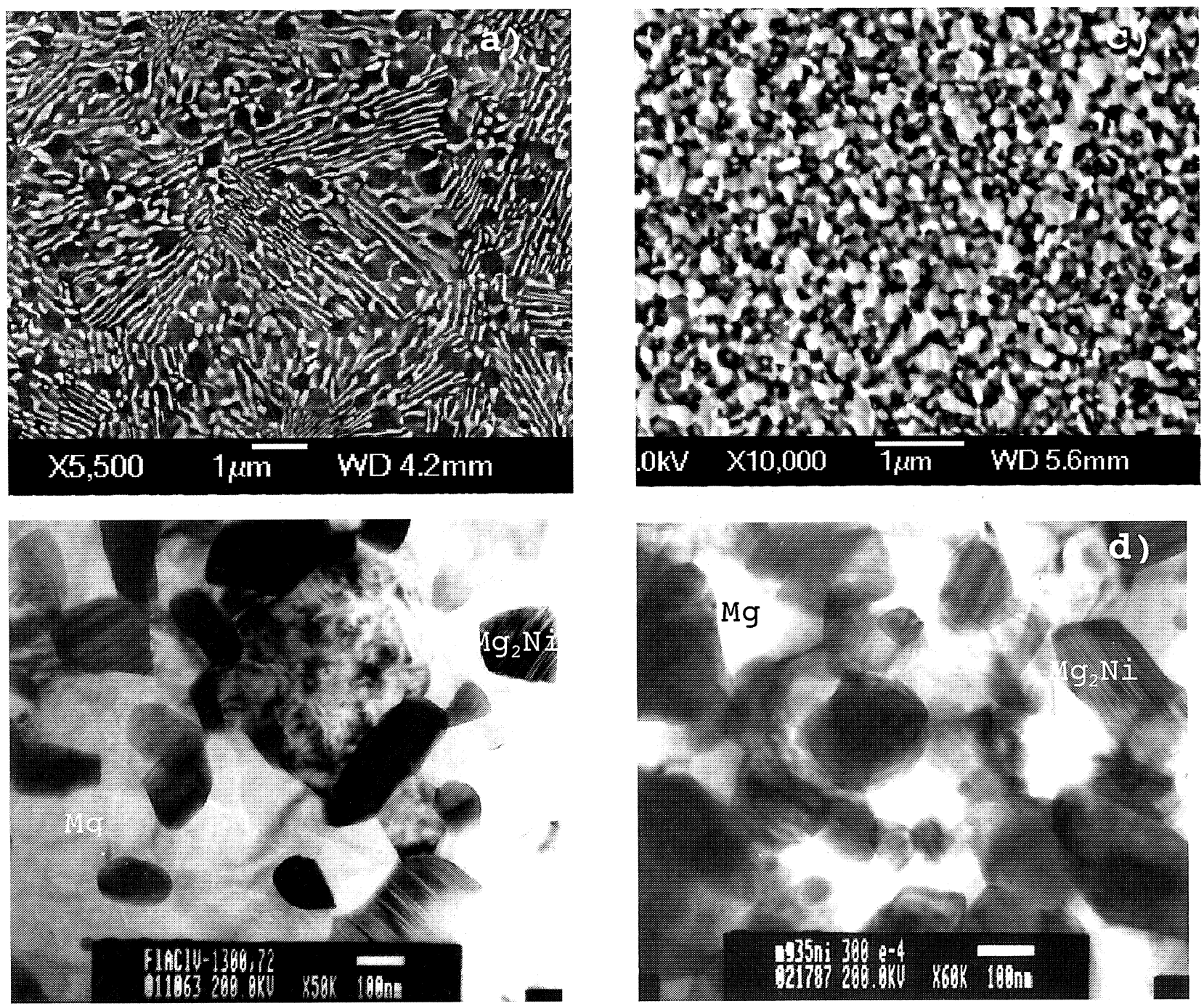

Figura 6. Microestructuras antes y después del ensayo a $300^{\circ} \mathrm{C}$. a) y b) $\mathrm{Mg} 23 \mathrm{Ni}$ c) y d) $\mathrm{Mg} 35 \mathrm{Ni}$.

Figure 6. Microstructures before and after testing at $300^{\circ} \mathrm{C}$. al and b) $\mathrm{Mg} 23 \mathrm{Nic}$ and d) $\mathrm{Mg} 35 \mathrm{Ni}$.

con $35 \%$ de Ni cristaliza en una sola etapa, a unos $170{ }^{\circ} \mathrm{C}$ si el calentamiento es isocrono, formándose las fases $\mathrm{Mg}_{2} \mathrm{Ni}$ y $\mathrm{Mg}$. En este estudio, se ha comprobado que la cinta $\mathrm{Mg} 35 \mathrm{Ni}$ ensayada a $100{ }^{\circ} \mathrm{C}$ estaba parcialmente cristalizada, apareciendo la fase $\mathrm{Mg}_{2} \mathrm{Ni}$, de unos $100 \mathrm{~nm}$ de tamaño, en una matriz amorfa. Por tanto, la ausencia de la fase metaestable $\mathrm{Mg}_{5,5} \mathrm{Ni}$ durante la cristalización de esta aleación y los valores de resistencia tan bajos que presenta concuerda con la hipótesis hecha anteriormente sobre el papel reforzante de la fase metaestable $\mathrm{Mg}_{5,5} \mathrm{Ni}$, en la aleación $\mathrm{Mg} 23 \mathrm{Ni}$. Los cambios de morfología y tamaño de las fases $\mathrm{Mg}$ y $\mathrm{Mg}_{2} \mathrm{Ni}$ que se producen en las dos aleaciones durante los ensayos a elevadas temperaturas, parecen influir en los procesos de deformación disminuyendo notablemente los valores de resistencia, aunque siempre son inferiores los de aleación con más ní- quel. Para comprobar esta hipótesis se está realizando en estas aleaciones cristalizadas un estudio sobre los mecanismos de deformación que tienen lugar a altas temperaturas.

\section{CONCLUSIONES}

- Los cambios microestructurales, cristalización y crecimiento de las fases de equilibrio, que experimentan los materiales desde el estado amorfo, por efecto del calentamiento hasta la temperatura de ensayo, provocan una evolución significativa en las propiedades mecánicas de ambos materiales.

- Los valores más elevados de resistencia mecánica los presentan las aleaciones en estado amorfo, alcanzando la aleación $\mathrm{Mg} 23 \mathrm{Ni}$, a $50^{\circ} \mathrm{C}$, valores de $450 \mathrm{MPa}$. 
- El inicio de la cristalización a $138^{\circ} \mathrm{C}$ en la aleación $\mathrm{Mg} 23 \mathrm{Ni}$ se caracteriza por la aparición de dos etapas elásticas. La presencia de la fase metaestable nanocristalina $\mathrm{Mg}_{5,5} \mathrm{Ni}$ le confiere al material elevada resistencia, que disminuye conforme se descompone en las fase de equilibrio eutéctica $\mathrm{Mg}+\mathrm{Mg}_{2} \mathrm{Ni}$.

- La aleación Mg35Ni muestra, en todo el intervalo de temperaturas estudiado, valores de resistencia mecánica y alargamiento más bajos que la aleación eutéctica. Estas diferencias se hacen más importantes a las temperaturas en las que se produce la cristalización, relacionándose con la ausencia en esta aleación de la fase metaestable $\mathrm{Mg}_{5,5} \mathrm{Ni}$.

\section{Agradecimientos}

Al Prof. Sommer del Max Planck Institute de Stuttgart por el suministro de los materiales y de la información sobre sus estudios de cristalización. Este trabajo está financiado por la Comunidad Autónoma de Madrid, Proyecto 07N/0074/2002.

\section{REFERENCIAS}

[1] I.J. Polmear, Magnesium and Magnesium alloys. ASM International, (1999), pp. 12

[2] S.G. Kim, A. InOUe y T. Masumoto, Mater. Trans. JIM 31(1990) 929-934.

[3] A. Inoue, A. Kato, T. Zhang , S.G. Kim y T. Masumoto, Mater. Trans. JIM 32 (1991) 609-616.

[4] T.W. Kempen, H. Nitsche H.F. SOMmer y E.J. MitTemeijer, Metall. Trans. 33 A (2002) 1.041-1.050.

[5] V. Rangelova y T. Spassov, J. Alloys Comp. 45 (2002)148-154.

[6] T. Spassov y V. Köster V, Z. Metallkd. 91 (2000) 675-679.

[7] N. CuI, P. He Y J.L. LuO, Acta Mater. 47 (1999) 3.737 3.743 .

[8] L.E.A. Berlouis, E. Cabrera, E. Hall-Barrientos, P. MaY, S. DodD, S. Morris y M.A. IMA, J. Alloys Comp. 305 (2000) 82-88.

[9] T. Spassov, P. SOlsona, S. Suriñach Y M.D. Baró, J. Alloys. Comp. 345 (2002) 123-129.

[10] L. Bakonyi, F. Mehener, M. Rapp, A. Cziraki, H. KRONMÜlleR y R. KiRCHHeIM, Z. Metallk 86 (1995) 619 621. 